\title{
Evaluation of Seed Quality Attributes of Sorghum Germplasm Accessions from Eastern, Coastal and Nyanza Regions, Kenya
}

\author{
C. W. Muui ${ }^{1}$, R. M. Muasya ${ }^{2}$, S. Nguluu $^{2}$ \& A. Kambura ${ }^{3}$ \\ ${ }^{1}$ School of Agriculture and Enterprise Development, Department of Agricultural Science and Technology, \\ Kenyatta University, P.O. Box 43844-00100, Nairobi, Kenya \\ ${ }^{2}$ School of Agriculture and Veterinary Sciences, Department of Dry Land Agriculture, South Eastern Kenya \\ University, P.O. Box 170-90200, Kitui, Kenya \\ ${ }^{3}$ School of Agriculture, Earth and Environmental Science, Taita Taveta, Voi, Kenya \\ Correspondence: C. W. Muui, School of Agriculture and Enterprise Development, Department of Agricultural \\ Science and Technology, Kenyatta University, P.O. Box 43844-00100, Nairobi, Kenya. E-mail: \\ catherinemuui@gmail.com
}

Received: March 25, 2020 Accepted: April 25, 2020 Online Published: May 8, 2020

doi:10.5539/sar.v9n3p9

URL: https://doi.org/10.5539/sar.v9n3p9

\begin{abstract}
Sorghum (Sorghum bicolor L.) is an important cereal crop in Kenya. Despite the crops importance, the yields attained by farmers in Eastern, Coastal and Nyanza regions of Kenya remain low. Access to good quality seeds of sorghum is one of the constraints facing the subsistence farmers. Good quality seed is important for increasing yield to attain food security. The aim of this study was to evaluate quality attributes of the seed used by farmers. A total of 108 germplasm accessions were obtained from 76 farmers. The seeds were tested for time and percentage of germination, seed vigour index, shoot and root dry weight. Data collected was subjected to analysis of variance. Means were separated using Fisher's Least Significance Difference test at $p \leq 0.05$. Seed samples of 26 accessions attained germination percentage below stipulated standards by Seeds and Plant Varieties Act CAP 326. Majority of seeds showed longer mean germination time with only nine accessions germinating in less than ten days. Seed vigour index was relatively high in most of the accessions, while biomass accumulation varied from high to very low among accessions. Though most of the seeds attained a high germination percentage, about $92 \%$ of seeds showed longer mean germination time. The environmental conditions in the fields, pre and post harvest handling practices impact on the seed quality hence the wide variability in germination percentage, germination time, seed vigour index and dry matter accumulation. Therefore the need to improve quality of seeds used by subsistence farmers by providing extension services on best pre and post harvest handling practices. Increasing production of sorghum in these regions will contribute significantly towards realizing food security. Further analysis could be carried out on genetic and sanitary quality aspects of the seeds planted by farmers in Eastern, Nyanza and Coastal regions.
\end{abstract}

Keywords: attributes, germplasm, seed quality, Sorghum bicolor

\section{Introduction}

Sorghum (Sorghum bicolor (L.) Moench) is an important cereal grain used and grown in semi arid areas as food for many families due to its nutritive value (Rao et al., 2016; FAO, 2018). The ability of sorghum to adapt to drought, salinity and high temperatures makes it a critical crop in the dry regions where other cereal crops produce low yields (Mamoudou, 2006). In Kenya, the crop is cultivated and highly consumed in semi-arid regions with low annual rainfall of about $300 \mathrm{~mm}$ which include Eastern $\left(1385 \mathrm{~m}\right.$ ASL, $76 \mathrm{~mm}$ month $^{-1}$ ), Coast $\left(185 \mathrm{~m} \mathrm{ASL}, 87 \mathrm{~mm}\right.$ month $\left.^{-1}\right)$ and Nyanza (1190m ASL, 130mm month $\left.{ }^{-1}\right)$ (Grieser et al., 2006). With its utilization closely related to maize, sorghum can be an alternative crop in marginal areas (Swigonova et al., 2004). The crop has got a large germplasm which could provide opportunities for a sustainable crop production for food security (Kange et al., 2014).

Sorghum production is mainly by subsistence farmers in marginalized regions in Kenya. Despite the many benefits associated with sorghum its production is still low (Muui et al., 2019). Farmers obtain seed from informal seed system which include retaining seed on-farm from previous harvests to plant the following season 
and farmer-to-farmer seed exchange networks (Ochieng et al., 2011; Muui et al., 2013, 2019). Where formal seed is available, the farmers cannot afford due to high seed prices. The informal seed supply system consists of farmer-managed seed production activities and is based on indigenous knowledge and local diffusion mechanisms. Maintaining crop production in terms of yield and quality grains which give the farmer maximum return requires good seed which carries the genetic, physiological, and physical quality aspects (Muasya et al., 2008; Ahmed et al., 2009). Good seed requires constant care to prevent loss of quality and to ensure high yield for farmers. Seed deterioration usually commences at physiological maturity and continues during harvest, processing and storage and is governed by the genetic constitution, environmental factors during seed development and storage conditions (McDonald, 1999; Muasya et al., 2008).

Seed quality is considered as an important factor for increasing yield unit area to attain food security (Badigannavar et al., 2016). Quality seeds have the ability for efficient utilization of available inputs such as fertilizers and moisture eventually maximizing yields (Jisha et al., 2013). Use of poor quality seed is one of the constraints to sorghum production in Kenya where majority of the farmers rely on the informal seed supply sources resulting to low yields (Ochieng et al., 2011). Poor seed quality at farm level is caused by poor drying of harvested grains, threshing practices, storage conditions (Harrison and Perry, 1976; Songa et al., 1995). Also, many subsistence farmers in Sub-Saharan Africa do not apply fertilizers to their farms (Jama et al., 1998; Ochieng et al., 2011; Muui et al., 2019). This is attributed to the fact that sorghum is often grown under marginal rainfall conditions and fertilizer prices are unfavourably high in relation to sorghum grain price. This practice of using little or no fertilizer affects both seed quality and yield of the crop negatively (Swinkels et al., 1997).

Sorghum landraces germplasm provides a great genetic variability with high preferences based on unique characteristics (Ng'uni et al., 2012). Sorghum being a food crop with the potential of alleviating the problem of food insecurity, there is need for using quality seeds for sustainable production by the subsistence farmers (Mwadalu and Mwangi, 2013). This study aimed at assessing the quality of on farm saved sorghum seeds used by farmers at eastern, coastal and nyanza regions of Kenya. A total of 108 germplasm accessions were collected from farmers to determine germination percentage, germination time, seed vigour index, seedling shoot and root dry weight.

\section{Materials and Methods}

\subsection{Description of experimental Site}

The experiment was carried out in Kenyatta University situated in Nairobi County about $20 \mathrm{Km}$ from Nairobi city along Nairobi-Thika road between August and October 2018. The county is characterized by a warm climate with temperatures varying between $12^{\circ} \mathrm{C}$ and $18.7^{\circ} \mathrm{C}$. The rainfall aggregate for the county is $1,000 \mathrm{~mm}$ per year. Its geographical coordinates are $1^{\circ} 10^{\prime} 0^{\prime \prime} \mathrm{S}, 36^{\circ} 50^{\prime} 0 " \mathrm{E}$ with an elevation of $1,720 \mathrm{~m}$ above sea level (ASL). The area has a bimodal rainfall pattern with an average of $1,000 \mathrm{~mm}$ per annum. The long rains occur between March and May while the short rains set in between October and December. The soils are acrisols, alisols, lixisols and luvisols (Shisanya et al., 2006).

\subsection{Experimental Treatments and Design}

\subsubsection{Experimental Layout and Data Collection}

The experiment was carried out in the laboratory and in a greenhouse. The two experiments were arranged in a Complete Randomised Design. A total of 108 sorghum germplasm accessions obtained from farmers comprising of 41 accessions from Eastern, 25 from Nyanza and 42 from Coastal regions of Kenya were used. The sorghum germplasm was collected from farmers in 2018 while conducting a baseline survey to assess the production systems for sorghum in the three regions (Muui et al., 2019).

\subsection{Crop Management and Data Collection}

Germination percentage and mean germination time experiments were carried out in the laboratory while seed vigour index, shoot and root dry weight was done in the greenhouse.

\subsubsection{Germination Percentage}

From each of the 108 sorghum germplasm accessions, a sample of 400 seeds was selected at random from the 1,000 -seed weight lot and grouped into four replicates of 100 seeds (ISTA, 2012). Each of the four replicates was placed in a germination tray with sterilized filter papers moistened with distilled water as a growth medium. The trays were illuminated with light during the whole period and temperatures maintained at $25 \pm 5^{\circ} \mathrm{C}$. Distilled water was added as necessary to maintain the correct moisture content. Germination count was done at the end of the fourth day and seedling evaluation at the end of the tenth day. Germination percentage was calculated as 
follows:

Germination percentage $(\%)=($ Number of seeds germinated/Number of seeds sown $) \times 100$

\subsubsection{Mean Germination Time (Days)}

The seeds used for testing germination percentage were also used in the determination of mean germination time. Emerged seedlings in each container were counted daily at an interval of 24 hours from the first day to the day no more germination occured. The mean germination time was calculated using the method described by Khan $e t$ al. (2010) as follows:

Mean germination time $=($ No. of germinated seedlings/Total no. of seeds sown $) \times$ Days after sowing

\subsubsection{Seed Vigour Index}

From the 108 sorghum germplasm accessions, a sample of 200 seeds was selected at random from 1,000-seed weight lot (same seed lot used for germination) and grouped into four replicates of 50 seeds. The fifty seeds were placed in plastic containers (pots) with sterilized forest soil as a growth medium for 21 days. Number of germinated seeds was recorded every 24 hours. Watering was done on daily basis and pots kept weed free throughout the experimental period. After 21 days, the seedlings were uprooted, soils washed off and a ruler used to measure the seedling height. Seed vigour index determination was done using the equation cited by Zhu et al., (2010) as follows:

Seed vigour index $(\mathrm{SVI})=$ Seedling height $\times \sum$ (number of germinated seedlings by end of test period/days after sowing)

\subsubsection{Shoot and Root Dry Weight (Grams)}

Seedlings used for seed vigour index were also used for dry weight measurement. A random sample of twenty five seedlings was taken and separated into shoot and root, dried in a forced-air oven at $72^{\circ} \mathrm{C}$ for 48 hours. The samples were fully dried such that no significant changes occurred before the tests were done. The dried shoots and roots were weighed using an electronic balance (model 6354) and recorded in grammes.

\subsection{Data Analysis}

The data collected on germination percentage, germination time, seed vigour index, shoot and root dry weight were managed in the Ms excel spreadsheet and subjected to one-way analysis of variance (ANOVA) using Statistical Analysis Software (SAS) version 9.1. Means were separated using Fisher's Least Significance Difference (LSD) test at $\mathrm{p} \leq 0.05$.

\section{Results}

\subsection{Germination Percentage}

There were statistical differences $(\mathrm{p} \leq 0.05)$ in the germination percentage of the germplasm accessions evaluated across the three regions of Kenya; Eastern (41), Nyanza (25) and coast (42). In the Eastern region, thirty-eight of the tested accessions had more than $70 \%$ while three accessions had less than $70 \%$ with local102 having the lowest germination (18.67\%) (Table 1). On the other hand, 29 of the tested accessions from coastal region had 70\% germination whereas 13 accessions showed less than $70 \%$ germination with the lowest, $18 \%$, from local accession gaddamssp38. In Nyanza, 15 of the tested accessions had 70\% germination. A relatively lower than $70 \%$ germination percentage was observed in ten germplasm accessions, but Ngware spp 2 recorded the lowest germination percentage of $18.0 \%$. 
Table 1. Germination percentages for sorghum germplasm accessions obtained from Eastern, Coastal and Nyanza regions in 2018

\begin{tabular}{|c|c|c|c|c|c|}
\hline \multicolumn{2}{|c|}{ Eastern region } & \multicolumn{2}{|c|}{ Coastal region } & \multicolumn{2}{|c|}{ Nyanza region } \\
\hline $\begin{array}{l}\text { Germplasm } \\
\text { accessions }\end{array}$ & $\begin{array}{l}\text { Germination } \\
(\%)\end{array}$ & $\begin{array}{l}\text { Germplasm } \\
\text { accessions }\end{array}$ & $\begin{array}{l}\text { Germination } \\
(\%)\end{array}$ & $\begin{array}{l}\text { Germplasm } \\
\text { accessions }\end{array}$ & $\begin{array}{l}\text { Germinatio } \\
\mathrm{n}(\%)\end{array}$ \\
\hline Local Variety68 & $97.67^{\mathrm{ab}}$ & Gadam Spp26 & $60.33^{\mathrm{ghi}}$ & Nyaktos sppl & $60.33^{\mathrm{fg}}$ \\
\hline Local Variety 69 & $84.67^{\text {cdefg }}$ & Gadam Spp27 & $66.67^{\mathrm{fgh}}$ & Ngware spp 2 & $66.67^{\mathrm{efg}}$ \\
\hline Local Variety70 & $77.67^{\text {fghi }}$ & Local Variety28 & $93.30^{\mathrm{ab}}$ & Ngware spp 3 & $18.00^{\mathrm{h}}$ \\
\hline Kivila Kyaivui71 & $84.33^{\text {cdefg }}$ & Local Variety29 & $33.33^{\mathrm{kl}}$ & $\mathrm{C}-26 \mathrm{spp} 4$ & $33.33^{\mathrm{h}}$ \\
\hline Kikomo72 & $93.33^{\mathrm{abcd}}$ & Mixed30 & $26.67^{\mathrm{kl}}$ & Nyadundo5 3 & $26.70^{\mathrm{h}}$ \\
\hline Rasta73 & $86.67^{\text {bcdef }}$ & Gadam Spp31 & $86.67^{\mathrm{abcd}}$ & Seredo6 & $86.67^{\mathrm{abc}}$ \\
\hline Kilala74 & $71.00^{\text {hij }}$ & Gadam Spp32 & $80.00^{\text {bcdef }}$ & Nyakabala $s p p 7$ & $80.00^{\text {bcde }}$ \\
\hline Local Variety75 & $71.00^{\text {hij }}$ & Gadam Spp33 & $86.67^{\mathrm{abcd}}$ & Nyaktos $s p p 8$ & $86.67^{\mathrm{abc}}$ \\
\hline Kitaa Kyaivui76 & $84.33^{\text {bcdefg }}$ & Gadam Spp34 & $58.00^{\mathrm{hi}}$ & Nyakabala $s p p 9$ & $58.00^{\mathrm{g}}$ \\
\hline Kari Mtama-177 & $95.67^{\mathrm{abc}}$ & Local Variety 35 & $29.00 \mathrm{kl}$ & Ngware spp10 & $29.00^{\mathrm{h}}$ \\
\hline Local Variety78 & $82.33^{\text {defgh }}$ & Gadam Spp36 & $86.30^{\mathrm{abcd}}$ & Ngware (white)spp 11 & $86.33^{\mathrm{abcd}}$ \\
\hline local Variety Red79 & $80.0^{\text {efgh }}$ & Kautimbi ssp37 & $93.30^{\mathrm{ab}}$ & Ngware spp12 & $93.33^{\mathrm{ab}}$ \\
\hline local variety 80 & $97.67^{\mathrm{ab}}$ & Gadam Spp38 & $18.00^{1}$ & Nyakabala $s p p 13$ & $18.00^{\mathrm{h}}$ \\
\hline Katengu81 & $100.00^{\mathrm{a}}$ & Gadam Spp39 & $97.67^{\mathrm{a}}$ & Nyakabala spp 14 & $97.67^{\mathrm{a}}$ \\
\hline local Brown82 & $100.00^{\mathrm{a}}$ & Kingundu $s p p 40$ & $95.33^{\mathrm{ab}}$ & Ochuti spp 15 & $95.33^{\mathrm{ab}}$ \\
\hline local Red83 & $86.67^{\text {abcdef }}$ & Local Variety41 & $100.00^{\mathrm{a}}$ & Nyakabala spp 16 & $100.00^{\mathrm{a}}$ \\
\hline Rasta spp84 & $100.00^{\mathrm{a}}$ & Local Variety 42 & $97.67^{\mathrm{a}}$ & Nyakabala spp 17 & $97.67^{\mathrm{a}}$ \\
\hline Muruge spp85 & $97.67^{\mathrm{ab}}$ & Gadam Spp43 & $93.00^{\mathrm{a}}$ & Othiwa spp 18 & $93.33^{\mathrm{ab}}$ \\
\hline Muveta spp86 & $100.00^{\mathrm{a}}$ & Local Variety44 & $95.33^{\mathrm{ab}}$ & Gadam Spp19 & $95.33^{\mathrm{ab}}$ \\
\hline Muvela spp87 & $100.00^{\mathrm{a}}$ & Local Variety 45 & $75.67^{\text {cdefg }}$ & Nyakabala $s p p 20$ & $75.67^{\text {cdef }}$ \\
\hline Mugeta spp88 & $100.00^{\mathrm{a}}$ & Gadam Spp46 & $100.00^{\mathrm{a}}$ & Andiwo spp21 & $100.00^{\mathrm{a}}$ \\
\hline Local Red89 & $66.67^{\mathrm{ij}}$ & Local Variety47 & $60.00^{\mathrm{ghi}}$ & Ngware spp 22 & $60.00^{\mathrm{fg}}$ \\
\hline Local Red90 & $100.00^{\mathrm{a}}$ & Local Variety 48 & $93.30^{\mathrm{ab}}$ & Oyundiwi-Jolejo23 & $93.33^{\mathrm{ab}}$ \\
\hline Ciumbichi91 & $71.00^{\text {hij }}$ & Local Variety 49 & $69.00^{\text {efgh }}$ & Seredo spp 24 & $69.00^{\text {efg }}$ \\
\hline Vaasya92 & $64.67^{\mathrm{j}}$ & Local Variety50 & $86.67^{\mathrm{abcd}}$ & Gadam Spp25 & $86.67^{\mathrm{abc}}$ \\
\hline local brown93 & $100.00^{\mathrm{a}}$ & KitaakyaIV VII51 & $87.00^{\mathrm{abcd}}$ & & \\
\hline Rasta94 & $97.67^{\mathrm{ab}}$ & Gadam Spp52 & $75.33^{\text {cdefg }}$ & & \\
\hline Muvuta spp95 & $91.3^{\text {abcde }}$ & Local Variety53 & $29.00^{\mathrm{kl}}$ & & \\
\hline Serena96 & $100.00^{\mathrm{a}}$ & Local Variety54 & $84.33^{\text {abcde }}$ & & \\
\hline Langi wa Mbesa97 & $100.00^{\mathrm{a}}$ & Kivilakyaivui55 & $93.00^{\mathrm{ab}}$ & & \\
\hline Rasta98 & $100.00^{\mathrm{a}}$ & Local Variety56 & $49.00^{\mathrm{ij}}$ & & \\
\hline Kaguru spp99 & $100.00^{\mathrm{a}}$ & Local Variety57 & $73.33^{\text {defgh }}$ & & \\
\hline Rasta spp100 & $97.67^{\mathrm{ab}}$ & Local Variety58 & $100.00^{\mathrm{a}}$ & & \\
\hline Light brown101 & $100.00^{\mathrm{a}}$ & Local Variety59 & $100.00^{\mathrm{a}}$ & & \\
\hline Local102 & $18.67^{\mathrm{k}}$ & Gadam Spp60 & $97.70^{\mathrm{a}}$ & & \\
\hline Local103 & $100.00^{\mathrm{a}}$ & Local Variety61 & $100.00^{\mathrm{a}}$ & & \\
\hline Repaeted104 Cultivar & $97.67^{\mathrm{ab}}$ & Local Variety62 & $97.70 \mathrm{a}$ & & \\
\hline Local brown105 & $86.67^{\text {bddef }}$ & Local Variety63 & $62.33^{\text {hgi }}$ & & \\
\hline Local Red106 & $100.00^{\mathrm{a}}$ & Local Variety64 & $95.67^{\mathrm{ab}}$ & & \\
\hline Local107 & $71.00^{\mathrm{hji}}$ & Local Variety65 & $75.66^{\text {cdefg }}$ & & \\
\hline Local Red108 & $73.00^{\text {hijg }}$ & KitaaKya iv vii66 & $91.33^{\mathrm{abc}}$ & & \\
\hline & & Ithaa67 & $37.66^{\mathrm{K}}$ & & \\
\hline LSD & 11.74 & & 16.24 & & 17.47 \\
\hline
\end{tabular}

Means followed by the same letter within the same column are not significantly different according to Fisher's Least Significance Difference (LSD) test at $\mathrm{p} \leq 0.05$.

\subsection{Mean Germination Time (Days)}

The mean germination time (MGT) revealed statistical differences $(\mathrm{p} \leq 0.05)$ for the germplasm accessions evaluated across the three regions: Eastern, Nyanza and Coast. From Eastern region, local red83, ciumbichi91and local102 had the shortest MGT of 8.40, while Katengu81, local variety 75, KitaaKyaivui 7, 
Rasta 73 and Rasta spp 100 had the longest MGT of 21.00 (Table 2). At the Coastal region, mixed30 and local variety35 accessions had the shortest MGT of 5.60 while, local variety28, localvariety41, localvariety44, kitaakyaIV VII51, Kitaa kya ivuii66 and Kavilakyaivui55 exhibited the longest MGT of 21.00 respectively (Table 2). In Nyanza Othiwa spp18, Nyakabala spp9 and Gadam spp25 had the shortest MGT of 11.20 while Ngware spp12, Ochutis spp15, Oyundiwi-Joleho23 and Serodo spp24 had the longest MGT of 21.00 respectively (Table 2).

Table 2. Mean germination time (days) of sorghum germplasm accessions obtained from Eastern, Coastal and Nyanza regions in 2018

\begin{tabular}{|c|c|c|c|c|c|}
\hline \multicolumn{2}{|l|}{ Eastern region } & \multicolumn{2}{|l|}{ Coastal region } & \multicolumn{2}{|l|}{ Nyanza region } \\
\hline $\begin{array}{l}\text { Germplasm } \\
\text { accessions }\end{array}$ & $\begin{array}{l}\text { MGT } \\
\text { (days) }\end{array}$ & $\begin{array}{l}\text { Germplasm } \\
\text { accessions }\end{array}$ & $\begin{array}{l}\text { MGT } \\
\text { (days) }\end{array}$ & $\begin{array}{l}\text { Germplasm } \\
\text { accessions }\end{array}$ & $\begin{array}{l}\text { MGT } \\
\text { (days) }\end{array}$ \\
\hline Local Variety68 & $16.80^{\mathrm{bcd}}$ & Gadam Spp26 & $18.20^{\mathrm{abc}}$ & Nyaktos $s p p l$ & $18.20^{\mathrm{abc}}$ \\
\hline Local Variety69 & $16.80^{\mathrm{bcd}}$ & Gadam Spp27 & $16.80^{\mathrm{bcd}}$ & Ngware spp2 & $19.60^{\mathrm{ab}}$ \\
\hline Local Variety70 & $14.00^{\mathrm{de}}$ & Local Variety28 & $21.00^{\mathrm{a}}$ & Ngware spp3 & $19.60^{\mathrm{ab}}$ \\
\hline Kivila Kyaivui71 & $18.20^{\mathrm{abc}}$ & Local Variety 29 & $19.60^{\mathrm{ab}}$ & $\mathrm{C}-26 \mathrm{spp} 4$ & $19.60^{\mathrm{ab}}$ \\
\hline $\begin{array}{l}\text { Kikomo72 } \\
\text { Kis }\end{array}$ & $18.20^{\mathrm{abc}}$ & Mixed30 & $5.60^{\mathrm{i}}$ & Nyadundo5 3 & $16.80^{\mathrm{bc}}$ \\
\hline Rasta73 & $21.00^{\mathrm{a}}$ & Gadam Spp31 & $16.80^{\mathrm{bcd}}$ & Seredo6 & $19.60^{\mathrm{ab}}$ \\
\hline Kilala74 & $16.80^{\mathrm{bcd}}$ & Gadam Spp32 & $18.20^{\mathrm{abc}}$ & Nyakabala $s p p 7$ & $15.40^{\mathrm{dc}}$ \\
\hline Local Variety75 & $21.00^{\mathrm{a}}$ & Gadam Spp33 & $16.80^{\mathrm{bcd}}$ & Nyaktos spp8 & $19.60^{\mathrm{ab}}$ \\
\hline Kitaa Kyaivui76 & $21.00^{\mathrm{a}}$ & Gadam Spp34 & $12.60^{\text {efg }}$ & Nyakabala $s p p 9$ & $11.20^{\mathrm{e}}$ \\
\hline Kari Mtama-177 & $18.20^{\mathrm{abc}}$ & Local Variety35 & $5.60^{\mathrm{i}}$ & Ngware sppl0 & $16.80^{\mathrm{bc}}$ \\
\hline Local Variety78 & $16.80^{\mathrm{bcd}}$ & Gadam Spp36 & $19.60^{\mathrm{ab}}$ & Ngware (white) spp 11 & $18.20^{\mathrm{abc}}$ \\
\hline local Variety Red79 & $18.20^{\mathrm{abc}}$ & Kautimbi ssp 37 & $19.60^{\mathrm{ab}}$ & Ngware spp12 & $21.00^{\mathrm{a}}$ \\
\hline local variety 80 & $15.40^{\text {cde }}$ & Gadam Spp38 & $8.40^{\mathrm{hi}}$ & Nyakabala spp 13 & $16.80^{\mathrm{bc}}$ \\
\hline Katengu81 & $21.00^{\mathrm{a}}$ & Gadam Spp39 & $18.20^{\mathrm{abc}}$ & Nyakabala spp 14 & $19.60^{\mathrm{ab}}$ \\
\hline local Brown82 & $19.60^{\mathrm{ab}}$ & Kingundu spp40 & $18.20^{\mathrm{abc}}$ & Ochuti spp 15 & $21.00^{\mathrm{a}}$ \\
\hline local Red83 & $8.40^{\mathrm{f}}$ & Local Variety 41 & $21.00^{\mathrm{a}}$ & Nyakabala spp 16 & $15.40^{\mathrm{dc}}$ \\
\hline Rasta spp84 & $15.40^{\text {cde }}$ & Local Variety42 & $18.20^{\mathrm{abc}}$ & Nyakabala spp 17 & $26.80^{\mathrm{bc}}$ \\
\hline Muruge spp85 & $19.60^{\mathrm{ab}}$ & Gadam Spp43 & $19.60^{\mathrm{ab}}$ & Othiwa spp 18 & $11.20^{\mathrm{e}}$ \\
\hline Muveta spp86 & $16.80^{\mathrm{bcd}}$ & Local Variety44 & $21.00^{\mathrm{a}}$ & Gadam Spp19 & $12.60^{\text {de }}$ \\
\hline Muvela spp87 & $16.80^{\mathrm{bcd}}$ & Local Variety45 & $9.80^{\mathrm{gh}}$ & Nyakabala spp 20 & $19.60^{\mathrm{ab}}$ \\
\hline Mugeta spp 88 & $16.80^{\mathrm{bcd}}$ & Gadam Spp46 & $18.20^{\mathrm{abc}}$ & Andiwo spp 21 & $15.40^{\mathrm{dc}}$ \\
\hline Local Red89 & $19.60^{\mathrm{ab}}$ & Local Variety 47 & $19.60^{\mathrm{ab}}$ & Ngware spp22 & $16.80^{\mathrm{bc}}$ \\
\hline Local Red90 & $19.60^{\mathrm{ab}}$ & Local Variety48 & $15.40^{\text {cde }}$ & Oyundiwi-Jolejo23 & $21.00^{\mathrm{a}}$ \\
\hline Ciumbichi91 & $8.40^{\mathrm{f}}$ & Local Variety49 & $16.80^{\mathrm{bcd}}$ & Seredo spp 24 & $21.00^{\mathrm{a}}$ \\
\hline Vaasya92 & $15.40^{\text {cde }}$ & Local Variety50 & $15.40^{\text {cde }}$ & Gadam Spp25 & $11.20^{\mathrm{e}}$ \\
\hline local brown93 & $19.60^{\mathrm{ab}}$ & KitaakyaIV VII51 & $21.00^{\mathrm{a}}$ & & \\
\hline Rasta94 & $16.80^{\mathrm{bcd}}$ & Gadam Spp52 & $18.20^{\mathrm{abc}}$ & & \\
\hline Muvuta spp95 & $16.80^{\mathrm{bcd}}$ & Local Variety53 & $8.40^{\mathrm{hi}}$ & & \\
\hline Serena96 & $16.80^{\mathrm{bcd}}$ & Local Variety54 & $18.20^{\mathrm{abc}}$ & & \\
\hline Langi wa Mbesa97 & $19.60^{\mathrm{ab}}$ & Kivilakyaivui55 & $21.00^{\mathrm{a}}$ & & \\
\hline Rasta98 & $21.00^{\mathrm{a}}$ & Local Variety56 & $8.40^{\text {hi }}$ & & \\
\hline Kaguru spp99 & $14.00^{\mathrm{de}}$ & Local Variety57 & $15.40^{\text {cde }}$ & & \\
\hline Rasta spp100 & $21.00^{\mathrm{a}}$ & Local Variety58 & $21.00^{\mathrm{a}}$ & & \\
\hline Light brown101 & $19.60^{\mathrm{ab}}$ & Local Variety59 & $19.60^{\mathrm{ab}}$ & & \\
\hline Local102 & $8.40^{\mathrm{f}}$ & Gadam Spp60 & $19.60^{\mathrm{ab}}$ & & \\
\hline Local103 & $19.60^{\mathrm{ab}}$ & Local Variety61 & $21.00^{\mathrm{a}}$ & & \\
\hline Repaeted104 Cultivar & $19.60^{\mathrm{ab}}$ & Local Variety62 & $15.40 \mathrm{cde}$ & & \\
\hline Local brown 105 & $12.60^{\mathrm{e}}$ & Local Variety63 & $12.60^{\text {def }}$ & & \\
\hline Local Red106 & $14.00^{\text {de }}$ & Local Variety64 & $16.80^{\mathrm{bcd}}$ & & \\
\hline Local107 & $12.60^{\mathrm{e}}$ & Local Variety65 & $11.20^{\mathrm{fgh}}$ & & \\
\hline Local Red108 & $15.40^{\text {cde }}$ & Kitaa Kya iv vii66 & $21.00^{\mathrm{a}}$ & & \\
\hline & & Ithaa67 & $14.00^{\mathrm{def}}$ & & \\
\hline LSD & 3.312 & & 2.98 & & 3.08 \\
\hline
\end{tabular}

Means followed by the same letter within the same column are not significantly different according to Fisher's Least Significance Difference (LSD) test at $\mathrm{p} \leq 0.05$. 


\subsection{Seed Vigour Index (SVI)}

Different germplasm accessions exhibited significant differences $(\mathrm{p} \leq 0.05)$ in seed vigour index (SVI) in Eastern, Nyanza and Coastal regions (Table 3). Local103 accession was superior with an SV1 0f 3499.3 from Eastern region, while local102 had the lowest SVI of 322.3. At Coastal region, SVI superiority was exhibited by accession localvariety 41 that recorded 4392.0 followed by the localvariety 42 with 4044.3 , while Gadam spp38 had the lowest SVI of 507.5. At Nyanza, Nyakabala spp 9 and Ochuti spp 15 were the superior accessions with a high SVI of 4080.9 and 4060.0 respectively, while Serodo Spp24 had the least SVI of 2119.8.

Table 3. Seed vigour index (SVI) for sorghum germplasm accessions obtained from Eastern, Coastal and Nyanza regions in 2018

\begin{tabular}{|c|c|c|c|c|c|}
\hline \multicolumn{2}{|c|}{ Eastern region } & \multicolumn{2}{|c|}{ Coastal region } & \multicolumn{2}{|c|}{ Nyanza region } \\
\hline $\begin{array}{l}\text { Germplasm } \\
\text { accessions }\end{array}$ & SVI & $\begin{array}{l}\text { Germplasm } \\
\text { accessions }\end{array}$ & SVI & $\begin{array}{l}\text { Germplasm } \\
\text { accessions }\end{array}$ & SVI \\
\hline Local Variety68 & $3196.6^{\mathrm{ab}}$ & Gadam Spp26 & $2759.2^{\text {hijk }}$ & Nyaktos sppl & $3570.8^{\text {abcde }}$ \\
\hline Local Variety69 & $2503.9^{\text {fgh }}$ & Gadam Spp27 & $1713.3^{\text {mnop }}$ & Ngware spp 2 & $3794.4^{\mathrm{abc}}$ \\
\hline Local Variety 70 & $1847.0^{\mathrm{jklm}}$ & Local Variety 28 & 3083.9defghi & Ngware spp3 & $3340.4^{\text {abcdef }}$ \\
\hline Kivila Kyaivui71 & $1287.2^{\text {no }}$ & Local Variety29 & $1271.5^{\text {nop }}$ & $\mathrm{C}-26 \mathrm{spp} 4$ & $3065.4^{\text {cdef }}$ \\
\hline Kikomo72 & $1644.5^{\operatorname{lmno}}$ & Mixed30 & $1321.2 \mathrm{n}^{\mathrm{op}}$ & Nyadundo5 3 & $2849.2^{\operatorname{defg}}$ \\
\hline Rasta73 & $2001.0^{\mathrm{jkl}}$ & Gadam Spp31 & 2994.5efghijk & Seredo6 & $3004.5^{\text {cdef }}$ \\
\hline Kilala74 & $1264.2^{\circ}$ & Gadam Spp32 & $2416.3^{\mathrm{jk} / \mathrm{m}}$ & Nyakabala spp7 & $3161.9^{\text {bcdef }}$ \\
\hline Local Variety75 & $1877.1^{\mathrm{jklm}}$ & Gadam Spp33 & $2689.6^{\mathrm{hijk}}$ & Nyaktos spp8 & $3028.1^{\text {cdef }}$ \\
\hline Kitaa Kyaivui76 & $2008.5^{\mathrm{ijkl}}$ & Gadam Spp34 & $1908.9^{\mathrm{lmn}}$ & Nyakabala $\operatorname{spp} 9$ & $4080.9^{\mathrm{a}}$ \\
\hline Kari Mtama-177 & $1977.6^{\mathrm{ijkl}}$ & Local Variety 35 & $624.7^{\mathrm{pq}}$ & Ngware spp10 & $3893.3^{\mathrm{ab}}$ \\
\hline Local Variety 78 & $1742.5^{\text {lmnop }}$ & Gadam Spp36 & $2823.6^{\text {ghijk }}$ & Ngware (white) spp 11 & $3499.2^{\text {abcde }}$ \\
\hline local Variety Red79 & $1767.7^{\mathrm{lmn}}$ & Kautimbi ssp37 & $3017.8^{\text {efghij }}$ & Ngware spp12 & $3351.1^{\text {abcdef }}$ \\
\hline local variety 80 & $2643.8^{\text {efgh }}$ & Gadam Spp38 & $507.5^{\mathrm{q}}$ & Nyakabala spp 13 & $3640.1^{\mathrm{abcd}}$ \\
\hline Katengu81 & $2613.3^{\text {efgh }}$ & Gadam Spp39 & $3864.7^{\mathrm{abc}}$ & Nyakabala spp 14 & $3270.6^{\text {abcdef }}$ \\
\hline local Brown82 & $2292.7^{\text {hij }}$ & Kingundu spp40 & $3224.0^{\text {cdefgh }}$ & Ochuti spp 15 & $4060.0^{\mathrm{a}}$ \\
\hline local Red83 & $2605.4^{\mathrm{efgh}}$ & Local Variety41 & $4392.0^{\mathrm{a}}$ & Nyakabala spp 16 & $3499.2^{\text {abcde }}$ \\
\hline Rasta spp84 & $1723.3^{\operatorname{lmnop}}$ & Local Variety42 & $4044.3^{\mathrm{ab}}$ & Nyakabala spp 17 & $3096.4^{\text {bcdef }}$ \\
\hline Muruge spp85 & $3036.0^{\text {abcde }}$ & Gadam Spp43 & $2907.5^{\text {fghijk }}$ & Othiwa spp 18 & $3536.5^{\text {abcde }}$ \\
\hline Muveta spp86 & $2960.0^{\text {bcdef }}$ & Local Variety44 & $3566.6^{\text {bcdefg }}$ & Gadam Spp 19 & $2769.1^{\mathrm{efg}}$ \\
\hline Muvela spp87 & $2273.3^{\text {hijk }}$ & Local Variety45 & $2787.5^{\text {hijk }}$ & Nyakabala spp 20 & $2906.9^{\operatorname{defg}}$ \\
\hline Mugeta spp 88 & $2666.7^{\text {defgh }}$ & Gadam Spp46 & $3781.7^{\mathrm{abcd}}$ & Andiwo spp21 & $3273.4^{\text {abcde }}$ \\
\hline Local Red89 & $1790.3^{\mathrm{klm}}$ & Local Variety 47 & $2248.4^{\mathrm{klm}}$ & Ngware spp 22 & $3485.5^{\text {abcde }}$ \\
\hline Local Red90 & $3271.0^{\mathrm{ab}}$ & Local Variety 48 & $3320.9^{\text {bcdefgh }}$ & Oyundiwi-Jolejo23 & $2615.8^{\mathrm{gf}}$ \\
\hline Ciumbichi91 & $2526.8^{\text {fgh }}$ & Local Variety49 & 2917.4fghik & Seredo spp 24 & $2119.8^{\mathrm{h}}$ \\
\hline Vaasya92 & $2823.7^{\text {bcdefg }}$ & Local Variety50 & $3299.1^{\text {bcdefgh }}$ & Gadam Spp25 & $13331.0^{\mathrm{h}}$ \\
\hline local brown 93 & $2978.7^{\text {bcdef }}$ & KitaakyaIV VII51 & $3619.2^{\text {bcdef }}$ & & \\
\hline Rasta94 & $2708.5^{\text {cdefg }}$ & Gadam Spp52 & $2607.3^{\mathrm{hijkl}}$ & & \\
\hline Muvuta spp95 & $3143.3^{\mathrm{abdc}}$ & Local Variety53 & $1107.5^{\text {opq }}$ & & \\
\hline Serena96 & $3167.0^{\mathrm{abcd}}$ & Local Variety54 & $2634.8^{\text {hijkl }}$ & & \\
\hline Langi wa Mbesa97 & $2286.0^{\mathrm{hij}}$ & Kivilakyaivui55 & $3174.4^{\text {ccdefghi }}$ & & \\
\hline Rasta98 & $2708.5^{\text {cdefg }}$ & Local Variety56 & $1321.3^{\text {nop }}$ & & \\
\hline Kaguru spp99 & $2275.0^{\text {hijk }}$ & Local Variety57 & $2783.1^{\text {hijk }}$ & & \\
\hline Rasta spp100 & $2677.0^{\text {defgh }}$ & Local Variety58 & $3299.1^{\text {bcdefgh }}$ & & \\
\hline Light brown 101 & $2405.6^{\text {ghi }}$ & Local Variety59 & $3545.7^{\text {bcdefg }}$ & & \\
\hline Local102 & $322.3^{\mathrm{p}}$ & Gadam Spp60 & $3610.9^{\text {bcdef }}$ & & \\
\hline Local103 & $3499.3^{\mathrm{a}}$ & Local Variety61 & $3131.7^{\text {cdefghi }}$ & & \\
\hline Repaeted104 Cultivar & $3201.6^{\mathrm{ab}}$ & Local Variety62 & 3198.3cdefgh & & \\
\hline Local brown 105 & $2406.5^{\mathrm{ghi}}$ & Local Variety63 & $2440.05^{\mathrm{ijklm}}$ & & \\
\hline Local Red106 & $1835.0^{\mathrm{jklm}}$ & Local Variety64 & $3736.3^{\text {abcde }}$ & & \\
\hline Local107 & $1854.7^{\mathrm{jklm}}$ & Local Variety65 & $3078.1^{\text {defghij }}$ & & \\
\hline Local Red108 & $1436.9^{\mathrm{mno}}$ & Kitaa Kya iv vii66 & $3289.8^{\text {bcdefgh }}$ & & \\
\hline & & Ithaa67 & $1378.6^{\text {nop }}$ & & \\
\hline LSD & 486.96 & & 758.15 & & 816.13 \\
\hline
\end{tabular}

Means followed by the same letter within the same column are not significantly different according to Fisher's Least Significance Difference (LSD) test at $p \leq 0.05$. 


\subsection{Shoot Dry Weight (SDW)}

There was a significant difference $(\mathrm{p} \leq 0.05)$ in shoot dry weight among various germplasm accessions from Eastern, Nyanza and Coastal regions (Table 4). In Eastern, Ciumbichi91 accession had the highest shoot dry weight of $0.046 \mathrm{~g}$, mixed30 accession from Coastal region was the leading in shoot dry weight of $0.073 \mathrm{~g}$ while, at Nyanza, Ochuti 15 was superior, recording $0.075 \mathrm{~g}$. Germplasm accessions with the least shoot dry weight were Gadam spp $27(0.0137 \mathrm{~g})$ and Seredo spp $24(0.0180 \mathrm{~g})$ from Coastal and Nyanza regions respectively.

Table 4. Shoot dry weight (SDW) (grams) of sorghum germplasm accessions obtained from Eastern, Coastal and Nyanza regions in 2018

\begin{tabular}{|c|c|c|c|c|c|}
\hline \multicolumn{2}{|c|}{ Eastern region } & \multicolumn{2}{|c|}{ Coastal region } & \multicolumn{2}{|c|}{ Nyanza region } \\
\hline Germplasm accessions & SDW $(\mathrm{g})$ & Germplasm accessions & SDW $(\mathrm{g})$ & Germplasm accessions & SDW $(\mathrm{g})$ \\
\hline Local Variety68 & $0.0241^{\text {detghi }}$ & Gadam Spp26 & $0.0470^{\text {bidefg }}$ & Nyaktos sppl & $0.0438^{\mathrm{etg}}$ \\
\hline Local Variety 69 & $0.0297^{\text {cde }}$ & Gadam Spp27 & $0.0137^{\mathrm{q}}$ & Ngware spp2 & $0.056^{\mathrm{bcd}}$ \\
\hline Local Variety70 & $0.0162^{\text {fghijkl }}$ & Local Variety28 & $0.0280^{\mathrm{klmnopq}}$ & Ngware spp3 & $0.0435^{\text {efgh }}$ \\
\hline Kivila Kyaivui71 & $0.0071^{\mathrm{lm}}$ & Local Variety29 & $0.0313^{\text {hijklmnop }}$ & $\mathrm{C}-26 \mathrm{spp} 4$ & $0.035^{\text {ghij }}$ \\
\hline Kikomo72 & $0.0122^{\mathrm{ijklm}}$ & Mixed30 & $0.0730^{\mathrm{a}}$ & Nyadundo5 3 & $0.035^{\text {fhij }}$ \\
\hline Rasta73 & $0.0320^{\mathrm{bcd}}$ & Gadam Spp31 & $0.0354^{\text {efghijklmnop }}$ & Seredo6 & $0.029^{\mathrm{ij}}$ \\
\hline Kilala74 & $0.0098^{\mathrm{jklm}}$ & Gadam Spp32 & $0.0234^{\text {nopq }}$ & Nyakabala $s p p 7$ & $0.059^{\mathrm{bc}}$ \\
\hline Local Variety 75 & $0.0260^{\text {defghi }}$ & Gadam Spp33 & $0.0450^{\text {bcdefg }}$ & Nyaktos spp8 & $0.044^{\text {defg }}$ \\
\hline Kitaa Kyaivui76 & $0.0181^{\text {efghijkl }}$ & Gadam Spp34 & $0.0290^{\mathrm{jklmnopq}}$ & Nyakabala $s p p 9$ & $0.054^{\text {bcde }}$ \\
\hline Kari Mtama-177 & $0.0235^{\text {defghij }}$ & Local Variety35 & $0.0220^{\mathrm{opq}}$ & Ngware spp10 & $0.045^{\mathrm{defg}}$ \\
\hline Local Variety78 & $0.0133^{\text {hijklm }}$ & Gadam Spp36 & $0.0290^{\mathrm{jklmnopq}}$ & Ngware (white)spp 11 & $0.064^{\mathrm{ab}}$ \\
\hline local Variety Red79 & $0.0173^{\text {efghijkl }}$ & Kautimbi ssp37 & $0.0340^{\text {fghijklmnop }}$ & Ngware spp 12 & $0.049^{\text {cdef }}$ \\
\hline local variety 80 & $0.0228^{\text {defghij }}$ & Gadam Spp38 & $0.0190^{\mathrm{qp}}$ & Nyakabala spp 13 & $0.053^{\text {bcde }}$ \\
\hline Katengu81 & $0.0207^{\text {defghijkl }}$ & Gadam Spp39 & $0.0470^{\text {bcdefg }}$ & Nyakabala spp 14 & $0.041^{\mathrm{fgh}}$ \\
\hline local Brown82 & $0.0190^{\text {defghijkl }}$ & Kingundu spp40 & $0.0289^{\text {jklmnopq }}$ & Ochuti spp 15 & $0.075^{\mathrm{a}}$ \\
\hline local Red83 & $0.0262^{\text {cdefgh }}$ & Local Variety41 & $0.0430^{\text {bcdefghilkl }}$ & Nyakabala spp 16 & $0.058^{\mathrm{bc}}$ \\
\hline Rasta spp84 & $0.01365^{\text {ghijkl }}$ & Local Variety 42 & $0.0360^{\text {efghijklmno }}$ & Nyakabala spp 17 & $0.048^{\text {cdef }}$ \\
\hline Muruge spp85 & $0.0263^{\text {cdefg }}$ & Gadam Spp43 & $0.0370^{\text {fghijklmnop }}$ & Othiwa spp 18 & $0.040^{\text {ghif }}$ \\
\hline Muveta spp86 & $0.0242^{\text {defghi }}$ & Local Variety44 & $0.0328^{\text {fghijklmnop }}$ & Gadam Spp 19 & $0.043^{\mathrm{efg}}$ \\
\hline Muvela spp87 & $0.0171^{\text {efghijkl }}$ & Local Variety45 & $0.0453^{\text {bcdefghi }}$ & Nyakabala spp20 & $0.031^{\text {ghij }}$ \\
\hline Mugeta spp88 & $0.0214^{\text {defghijk }}$ & Gadam Spp46 & $0.0540^{\mathrm{bcd}}$ & Andiwo spp21 & $0.034^{\text {ghij }}$ \\
\hline Local Red89 & $0.0301^{\text {cde }}$ & Local Variety47 & $0.0500^{\mathrm{bc}}$ & Ngware spp 22 & $0.038^{\text {fghi }}$ \\
\hline Local Red90 & $0.0230^{\text {defghij }}$ & Local Variety 48 & $0.0045^{\text {bcdefghij }}$ & Oyundiwi-Jolejo23 & $0.026^{\mathrm{jk}}$ \\
\hline Ciumbichi91 & $0.0460^{\mathrm{a}}$ & Local Variety 49 & $0.0510^{\text {bcde }}$ & Seredo spp 24 & $0.018^{\mathrm{k}}$ \\
\hline Vaasya92 & $0.0450^{\mathrm{ab}}$ & Local Variety50 & $0.0430^{\text {bcdefghijkl }}$ & Gadam Spp 25 & $0.036^{\text {ghij }}$ \\
\hline local brown93 & $0.0274^{\text {cdefg }}$ & KitaakyaIV VII51 & $0.0560^{\mathrm{b}}$ & & \\
\hline Rasta94 & $0.0230^{\text {defghij }}$ & Gadam Spp52 & $0.0456^{\text {bcdefgi }}$ & & \\
\hline Muvuta spp95 & $0.038^{\mathrm{abc}}$ & Local Variety53 & $0.0390^{\text {bcdefghijklm }}$ & & \\
\hline Serena96 & $0.0297^{\text {cdef }}$ & Local Variety54 & $0.0260^{\mathrm{mnopq}}$ & & \\
\hline Langi wa Mbesa97 & $0.0200^{\text {defghijkl }}$ & Kivilakyaivui55 & $0.03832^{\text {cdefghijklm }}$ & & \\
\hline Rasta98 & $0.0230^{\text {defghij }}$ & Local Variety56 & $0.02113^{\mathrm{qp}}$ & & \\
\hline Kaguru spp99 & $0.0180^{\text {efghijkl }}$ & Local Variety57 & $0.0517^{\text {bcde }}$ & & \\
\hline Rasta spp100 & $0.0237^{\text {defghi }}$ & Local Variety58 & $0.0330^{\text {fghijklmnop }}$ & & \\
\hline Light brown101 & $0.0216^{\text {defghijk }}$ & Local Variety59 & $0.0382^{\text {defghijklm }}$ & & \\
\hline Local102 & $0.0088^{\mathrm{klm}}$ & Gadam Spp60 & $0.0452^{\text {bcdefghij }}$ & & \\
\hline Local103 & $0.0280^{\text {cdef }}$ & Local Variety61 & $0.0280^{\mathrm{klmnopq}}$ & & \\
\hline Repaeted104 Cultivar & $0.0260^{\text {cdefg }}$ & Local Variety62 & $0.0271^{\mathrm{mnopq}}$ & & \\
\hline Local brown 105 & $0.0200^{\text {defghijkl }}$ & Local Variety63 & $0.0380^{\text {defghijklm }}$ & & \\
\hline Local Red106 & $0.0120^{\mathrm{ijklm}}$ & Local Variety64 & $0.0440^{\text {bcdefghij }}$ & & \\
\hline Local107 & $0.0163^{\text {fghijkl }}$ & Local Variety65 & $0.0490^{\text {bcde }}$ & & \\
\hline Local Red108 & $0.0144^{\text {ghijkl }}$ & Kitaa Kya iv vii66 & $0.0487^{\text {bcdef }}$ & & \\
\hline & & Ithaa67 & $0.0320^{\text {ghijklmnop }}$ & & \\
\hline LSD & 0.014 & & 0.016 & & 0.011 \\
\hline
\end{tabular}

Means followed by the same letter within the same column are not significantly different according to Fisher's Least Significance Difference (LSD) test at $\mathrm{p} \leq 0.05$.

\subsection{Root Dry Weight (RDW) (Grams)}

Root dry weight exhibited significant differences $(\mathrm{p} \leq 0.05)$ among germplasm accessions from Eastern, Coastal 
and Nyanza regions (Table 5). Vaasya92 (0.0152g), Nyakabala spp9 $(0.0188 \mathrm{~g})$ and local variety45 (0.039g) from Eastern, Nyanza and Coastal regions respectively had more root dry weight. Kilala74 in Eastern recorded the least root dry weight of $0.0020 \mathrm{~g}$ while in Nyanza Seredo spp 24 had the least weight of $0.0036 \mathrm{~g}$.

Table 5. Root dry weight (RDW) (grams) of sorghum germplasm accessions obtained from Eastern, Coastal and Nyanza regions in 2018

\begin{tabular}{|c|c|c|c|c|c|}
\hline \multicolumn{2}{|c|}{ Eastern region } & \multicolumn{2}{|c|}{ Coastal region } & \multicolumn{2}{|c|}{ Nyanza region } \\
\hline $\begin{array}{l}\text { Germplasm } \\
\text { accessions }\end{array}$ & $\begin{array}{l}\text { RDW } \\
(\mathrm{g})\end{array}$ & $\begin{array}{l}\text { Germplasm } \\
\text { accessions }\end{array}$ & $\begin{array}{l}\text { RDW } \\
(\mathrm{g})\end{array}$ & $\begin{array}{l}\text { Germplasm } \\
\text { accessions }\end{array}$ & $\begin{array}{l}\text { RDW } \\
(\mathrm{g})\end{array}$ \\
\hline Local Variety68 & $0.0052^{\mathrm{j} k l m n}$ & Gadam Spp26 & $0.014 \mathrm{c}$ & Nyaktos $s p p 1$ & $0.0074^{\mathrm{dc}}$ \\
\hline Local Variety69 & $0.0121^{\mathrm{abcd}}$ & Gadam Spp27 & $0.004 \mathrm{c}$ & Ngware spp2 & $0.0070^{\mathrm{dc}}$ \\
\hline Local Variety70 & $0.0056^{\text {hijklmn }}$ & Local Variety 28 & $0.006 \mathrm{c}$ & Ngware spp3 & $0.0053^{\text {def }}$ \\
\hline Kivila Kyaivui71 & $0.0024^{\mathrm{mnop}}$ & Local Variety29 & $0.009^{c}$ & $\mathrm{C}-26 \mathrm{spp} 4$ & $0.0081^{\mathrm{dc}}$ \\
\hline Kikomo72 & $0.0037^{\mathrm{Imnop}}$ & Mixed30 & $0.010^{\mathrm{c}}$ & Nyadundo5 3 & $0.0052^{\mathrm{def}}$ \\
\hline Rasta73 & $0.0037^{\mathrm{Imnop}}$ & Gadam Spp31 & $0.010^{\mathrm{c}}$ & Seredo6 & $0.0060^{\text {dce }}$ \\
\hline Kilala74 & $0.0020^{\text {no }}$ & Gadam Spp32 & $0.007^{\mathrm{c}}$ & Nyakabala $s p p 7$ & $0.0068^{\mathrm{dce}}$ \\
\hline Local Variety75 & $0.0102^{\text {bdefg }}$ & Gadam Spp33 & $0.0077^{\mathrm{c}}$ & Nyaktos $s p p 8$ & $0.0051^{\text {def }}$ \\
\hline Kitaa Kyaivui76 & $0.0060^{\text {ghijklm }}$ & Gadam Spp34 & $0.014^{\mathrm{c}}$ & Nyakabala $s p p 9$ & $0.0188^{\mathrm{a}}$ \\
\hline Kari Mtama-177 & $0.0039^{1 \mathrm{mnop}}$ & Local Variety 35 & $0.005^{\mathrm{c}}$ & Ngware spp10 & $0.0071^{\mathrm{dc}}$ \\
\hline Local Variety78 & $0.0053^{\text {hijlkmn }}$ & Gadam Spp36 & $0.006^{\mathrm{c}}$ & Ngware (white)spp 11 & $0.0080^{\mathrm{dc}}$ \\
\hline local Variety Red79 & $0.0026^{\text {mnop }}$ & Kautimbi ssp37 & $0.010^{\mathrm{c}}$ & Ngware spp12 & $0.0072^{\mathrm{dc}}$ \\
\hline local variety 80 & $0.0087^{\text {cdefghij }}$ & Gadam Spp38 & $0.0004^{c}$ & Nyakabala spp 13 & $0.00706^{\mathrm{dc}}$ \\
\hline Katengu 81 & $0.0058^{\text {ghijklm }}$ & Gadam Spp39 & $0.016 b c$ & Nyakabala spp 14 & $0.0073^{\mathrm{dc}}$ \\
\hline local Brown82 & $0.01110^{\text {abcde }}$ & Kingundu spp 40 & $0.007^{\mathrm{c}}$ & Ochuti spp 15 & $0.0120^{\mathrm{b}}$ \\
\hline local Red83 & $0.00508^{\text {ghijklm }}$ & Local Variety 41 & $0.011^{\mathrm{c}}$ & Nyakabala spp 16 & $0.0091^{\mathrm{c}}$ \\
\hline Rasta spp84 & $0.0125^{\mathrm{abc}}$ & Local Variety 42 & $0.117^{\mathrm{c}}$ & Nyakabala spp 17 & $0.0080^{\mathrm{dc}}$ \\
\hline Muruge spp85 & $0.0110^{\text {abcde }}$ & Gadam Spp43 & $0.010^{\mathrm{c}}$ & Othiwa spp 18 & $0.0068^{\mathrm{dc}}$ \\
\hline Muveta spp86 & $0.0097^{\text {bdefg }}$ & Local Variety 44 & $0.009^{c}$ & Gadam Spp 19 & $0.007^{\mathrm{dc}}$ \\
\hline Muvela spp87 & $0.0045^{\mathrm{jklmn}}$ & Local Variety45 & $0.039^{\mathrm{a}}$ & Nyakabala spp20 & $0.0033^{\mathrm{f}}$ \\
\hline Mugeta spp88 & $0.0065^{\text {fghijklm }}$ & Gadam Spp46 & $0.011^{\mathrm{c}}$ & Andiwo spp 21 & $0.0071^{\mathrm{dc}}$ \\
\hline Local Red89 & $0.0106^{\text {bcdef }}$ & Local Variety 47 & $0.014^{\mathrm{c}}$ & Ngware spp 22 & $0.008^{\mathrm{dc}}$ \\
\hline Local Red90 & $0.0049^{\mathrm{ijk} k \mathrm{mn}}$ & Local Variety 48 & $0.010^{\mathrm{c}}$ & Oyundiwi-Jolejo23 & $0.0038^{\mathrm{ef}}$ \\
\hline Ciumbichi91 & $0.0058^{\text {hijklmn }}$ & Local Variety 49 & $0.014^{\mathrm{c}}$ & Seredo spp 24 & $0.0036^{\mathrm{f}}$ \\
\hline Vaasya92 & $0.0152^{\mathrm{a}}$ & Local Variety50 & $0.007^{\mathrm{c}}$ & Gadam Spp25 & $0.0089^{\mathrm{c}}$ \\
\hline local brown 93 & $0.0029^{\mathrm{mnop}}$ & KitaakyaIV VII51 & $0.015^{\mathrm{c}}$ & & \\
\hline Rasta94 & $0.0085^{\text {cdefghijk }}$ & Gadam Spp52 & $0.009^{c}$ & & \\
\hline Muvuta spp95 & $0.0065^{\text {ghijklm }}$ & Local Variety53 & $0.010^{\mathrm{c}}$ & & \\
\hline Serena96 & $0.0079^{\text {defghijkl }}$ & Local Variety54 & $0.006^{\mathrm{c}}$ & & \\
\hline Langi wa Mbesa97 & $0.0048^{\mathrm{ijklmn}}$ & Kivilakyaivui55 & $0.010^{\mathrm{c}}$ & & \\
\hline Rasta98 & $0.0085^{\text {cdefghijk }}$ & Local Variety56 & $0.010^{c}$ & & \\
\hline Kaguru spp99 & $0.0054^{\mathrm{ijk} k \mathrm{mn}}$ & Local Variety57 & $0.012^{\mathrm{c}}$ & & \\
\hline Rasta spp100 & $0.0106^{\text {bcdef }}$ & Local Variety58 & $0.007^{\mathrm{c}}$ & & \\
\hline Light brown101 & $0.0045^{\mathrm{klmnop}}$ & Local Variety59 & $0.008^{c}$ & & \\
\hline Local102 & $0.0034^{\mathrm{mno}}$ & Gadam Spp60 & $0.011^{\mathrm{c}}$ & & \\
\hline Local103 & $0.0138^{\mathrm{ab}}$ & Local Variety61 & $0.005^{\mathrm{c}}$ & & \\
\hline $\begin{array}{l}\text { Repaeted104 } \\
\text { Cultivar }\end{array}$ & $0.0058^{\text {hijklmn }}$ & Local Variety62 & $0.006^{\mathrm{c}}$ & & \\
\hline Local brown 105 & $0.0090^{\text {cdefghi }}$ & Local Variety63 & $0.011^{\mathrm{c}}$ & & \\
\hline Local Red106 & $0.0030^{\mathrm{mnop}}$ & Local Variety64 & $0.031^{\mathrm{ab}}$ & & \\
\hline Local107 & $0.0067^{\text {ghijklm }}$ & Local Variety65 & $0.014^{\mathrm{c}}$ & & \\
\hline Local Red108 & $0.0035^{\operatorname{lmnop}}$ & Kitaa Kya iv vii66 & $0.010^{\mathrm{c}}$ & & \\
\hline & & Ithaa67 & $0.012^{\mathrm{c}}$ & & \\
\hline LSD & 0.005 & & 0.015 & & 0.003 \\
\hline
\end{tabular}

Means followed by the same letter within the same column are not significantly different according to Fisher's Least Significance Difference (LSD) test at $\mathrm{p} \leq 0.05$. 


\section{Discussions}

In this study, there was a high variability in the germination percentages for the sorghum seeds obtained from Eastern, Coastal and Nyanza regions of Kenya. A majority of the seeds exhibited a high germination percentage of $70 \%$ which is within the set standards by the Seeds and Plant Varieties Act of CAP 326 of the Kenyan constitution for sorghum varieties. However, part of the germplasm had low germination percentages indicating presence of low quality seeds used by farmers. Previous studies have reported that farmers obtain sorghum seeds from previously saved seeds, local markets, borrow from neighbors (Ochieng et al., 2011; Catherine et al., 2013; Kange et al., 2014; Muui et al., 2019). Majority of subsistence farmers in semi arid areas produce crops without fertilizers (Jama et al., 1998; Muui et al., 2013). Results of a baseline survey assessing production systems at coastal, Nyanza and eastern regions revealed that most farmers do not use fertilizers (Muui et al., 2019). This results to low yields and poor quality seeds since most soil nutrients have already been depleted (Songa et al., 1994; Craine et al., 2018). High humidity under elevated temperatures in these regions may have contributed to rapid deterioration of the seeds. There is evidence that elongated exposure to high temperatures and moisture would significantly reduce seed germination potential in many crops (Nagel et al., 2016). Besides, post-harvest seed handling and packaging also influence the rate of seed deterioration and hence has a direct impact on seed germination potential (Kange et al., 2014). Studies conducted in eastern, coast and Nyanza reported that most farmers have low education and therefore do not understand the best agronomic and post harvest handling practices which could help increase the quality of seed (Muui et al., 2013; 2019).

The mean time of germination for a seed indicates the time taken by a seed to develop critical structures crucial for germination success, survival, and for faster and uniform establishment. In this study, the mean germination time observed ranged from short, moderate to long. The shorter the mean germination time, the greater the seed vigour. A prolonged MGT may be an indication of deteriorated seed quality as a result of exposure of the seeds to harsh or unfavourable conditions in the field and after harvesting (Bewley and Black, 2012). Such conditions slows the rate of emergence and growth of the seedlings (Amirmoradi and Feizi, 2017); and eventually limits the seedlings from taking advantage of the available nutrients and resources for maximum yield within a short time (Bradford, 2002).

The seed vigour index variation in this study could be attributed to the diverse conditions during production, source of seeds and post harvest handling practices. Increased seedling vigour is an indication of effective germination, seedling emergence early seedling growth and improved grain yield (Lamichhane et al., 2018). Harsh conditions during the late stage of seed development might have also contributed to hormonal imbalance within the seeds which promotes physiological seed dormancy (Cotado and Munné-Bosch, 2020). Longer storage is associated with deterioration of seed stored microRNAs and other important proteins that have a role in the maintenance of high seed viability (Sahu et al., 2017; Sano and Rajjou, 2020).

The shoot biomass of seedlings varied from high to low attributed to pre and post harvest handling practices. A high shoot biomass is an indicator of increased seed vigour and subsequent crop growth cycles (Maucieri et al., 2016). Rapid development of the shoot is associated with the development of more leaves, which is important for the interception of photosynthetic active radiation that enhances rates of photosynthesis resulting in high biomass accumulation (Ceotto et al., 2013). Furthermore, genotypes with higher shoot dry matter have the capability of withstanding drought due to improved water and nutrient use efficiency (Verma et al., 2018). Majority of the germplasm displayed moderate to low root dry matter. Low root biomass has been reported to be as a result of reduced root growth in germplasm consisting of low quality seeds (Joshi et al., 2017). According to Blaha and Pazderu (2013), high seed quality leads to development of seedling with roots of greater biomass an indication of ability to withstand stress condition and also facilitate formation of high quality grains for the subsequent generations.

\section{Conclusion and Recommendations}

The ability of sorghum to perform well in semi arid areas makes it an important cereal crop to achieve food security. Farmers in Eastern, Nyanza and Coastal regions plant sorghum seeds obtained from diverse informal sources. The environmental conditions in the field and, pre and post harvest handling practices impact on the seed quality hence the wide variability in germination percentage, germination time, seed vigour index and dry matter accumulation in seedlings. This shows the need to improve and monitor the quality of seeds used by subsistence farmers. The quality of sorghum seeds could be improved by providing extension services on best pre and post harvest handling practices. Increasing production of sorghum in these regions will contribute significantly towards realizing food security. Further analysis could be carried out on genetic and sanitary quality aspects of the seeds planted by farmers in Eastern, Nyanza and Coastal regions. 


\section{Acknowledgements}

The authors acknowledge farmers from Eastern, Nyanza and Coastal regions of Kenya for providing sorghum germplasm accessions and the County agricultural officers for allowing access to the regions. National Research Fund (NRF), Kenya is gratefully acknowledged for funding this study.

\section{References}

Ahmed, H. M. I., Gregg, B. R., \& Louwaars, N. P. (2009). Seed Systems for Underutilized crops. Acta Horticulture (ISHS), 806, 459-464. https://doi.org/10.17660/ActaHortic.2009.806.57

Amirmoradi, S., \& Feizi, H. (2017). Can mean germination time predict seed vigor of canola (Brassica napu L.) seed lots? Acta Agrobotanica, 70(4), 1729. https://doi.org/10.5586/aa.1729

Badigannavar, A., Girish, G., Ramachandran, V., \& Ganapathi, T. R. (2016). Genotypic variation for seed protein and mineral content among post-rainy season-grown sorghum genotypes. The Crop Journal, 4(1), 61-67, https://doi.org/10.1016/j.cj.2015.07.002

Bewley, J. D., \& Black, M. (2012). Physiology and biochemistry of seeds in relation to germination: volume 2: viability, dormancy, and environmental control. Springer Science and Business Media.

Blaha, L., \& Pazderu, K. (2013). Influence of the root and seed traits on tolerance to abiotic stress. In Agricultural Chemistry. IntechOpen. https://doi.org/10.5772/55656

Bradford, K. J. (2002). Applications of hydrothermal time to quantifying and modeling seed germination and dormancy. Weed Science, 50(2), 248-260. https://doi.org/10.1614/0043-1745(2002)050[0248:AOHTTQ]2.0.CO;2

Catherine, W. M., Reuben, M. M., \& Duncan, T. K. (2013). Identification and evaluation of sorghum (Sorghum bicolor (L.) moench) germplasm from Eastern Kenya. African Journal of Agricultural Research, 8(37), 4573-4579.

Ceotto, E., Di Candilo, M., Castelli, F., Badeck, F. W., Rizza, F., Soave, C., \& Marletto, V. (2013). Comparing solar radiation interception and use efficiency for the energy crops giant reed (Arundo donax L.) and sweet sorghum (Sorghum bicolor L. Moench). Field Crops Research, 149, 159-166. https://doi.org/10.1016/j.fcr.2013.05.002

Cotado, A., Garcia, M. B., \& Munné-Bosch, S. (2020). Physiological seed dormancy increases at high altitude in Pyrenean saxifrage (Saxifraga longifolia Lapeyr.). Environmental and Experimental Botany, 171(August 2019), 103929. https://doi.org/10.1016/j.envexpbot.2019.103929

Craine, J. M., Elmore, A. J., Wang, L., Aranibar, J., Bauters, M., Boeckx, P., \& Fang, Y. (2018). Isotopic evidence for oligotrophication of terrestrial ecosystems. Nature Ecology and Evolution, 2(11), 1735. https://doi.org/10.1038/s41559-018-0694-0

FAO. (2018). Food and Agricultural Organization of the United Nations Faostatstatistics database. Retrieved from http://www.fao.org/faostat/en/\#data/QCProductionofselectedcerealcrops

Grieser, J. (2006). The FAO local climate estimator. Environment and Natural Resources Service Working Paper No. 9. Food and Agriculture Organization 2006. Retrieved from www.fao.org/sd/2002/en1203a_en.htm

Jama, B., Amandou, I., Amadalo, B., Wolf, J., Rao, M. R., \& Buresh, R. J. (1998). The Potential of Improved Fallows to Improve and Conserve the Fertility of Nutrients-Depleted Soils of Western Kenya. Agricultural Research and Development for Sustainable Resource Management and Increased Production. Proceedings of the 6th biennial KARI scientific conference. Retrieved from https://inis.iaea.org/search/search.aspx?orig_q=RN:38037858

Jisha, K. C., Vijayakumari, K., \& Puthur, J. T. (2013). Seed priming for abiotic stress tolerance: an overview. Acta Physiologiae Plantarum, 35(5), 1381-1396. https://doi.org/10.1007/s11738-012-1186-5

Joshi, D. C., Singh, V., Hunt, C., Mace, E., van Oosterom, E., Sulman, R., \& Hammer, G. (2017). Development of a phenotyping platform for high throughput screening of nodal root angle in sorghum. Plant methods, 13(1), 56. https://doi.org/10.1186/s13007-017-0206-2

Kange, A. M., Cheruiyot E. K., Ogendo, J. O., Arama, P. F., \& Ochola, S. O. (2014). Pre- and post harvest factors affecting sorghum production (Sorghum bicolor L. Moench) among smallholder farming Communities. International Journal of Agronomy and Agricultural Research, 5(4), 40-47.

https://doi.org/10.1186/s40066-015-0034-4 
Khan, A. Z., Shah, P., Mohd, F., \& Zubair, M. (2010). Vigour tests used to rank seed lot quality and predict field emergence in Wheat, Pakistan Journal of Botany, 3147-3155. https://www.researchgate.net/publication/287739275pdf

Lamichhane, J. R., Debaeke, P., Steinberg, C., You, M. P., Barbetti, M. J., \& Aubertot, J. N. (2018). Abiotic and biotic factors affecting crop seed germination and seedling emergence: a conceptual framework. Plant and soil, 1-28. https://doi.org/10.1007/s11104-018-3780-9

Mamoudou, H. D., Hurry, G., Alfred, S., Alphons, G. J., \& Van, B. (2006). Sorghum grain as human food in Africa: relevance of content of starch and amylase activities. African Journal Biotechnology, 5, 384-395. Retrieved from http://www.academicjournals.org/AJB

Maucieri, C., Cavallaro, V., Caruso, C., Borin, M., Milani, M., \& Barbera, A. (2016). Sorghum biomass production for energy purpose using treated urban wastewater and different fertilization in a Mediterranean environment. Agriculture, 6(4), 67. https://doi.org/10.3390/agriculture6040067

McDonald, M. B. (1999). Seed deterioration: physiological, repair and assessment. Journal of Seed Science and Technology, 27, 177-237.

Muasya, R. M., Lommen, W. J. M., Muui, C. W., \& Struik, P. C. (2008). How weather during development of common bean (Phaseolus vulgaris L.) affects the crop's Maximum attainable seed quality. NJAS-Wageningen Journal of Life Sciences, 56, 85-100. https://doi.org/10.1016/S1573-5214(08)80018-8

Muui, C. W, Muasya, R. M., Nguluu, S., Kambura, A, Kathuli, P., Mweu, B., \& Odhiambo, D. O. (2019). Sorghum Landraces Production Practices in Nyanza, Coast andEastern Regions, Kenya. Journal of Economics and Sustainable Development, 10(10).

Muui, C. W., Muasya, R. M., \& Kirubi, D. T. (2013). Baseline survey on factors affecting sorghum production and use in eastern Kenya. African Journal of Food, Agriculture Nutrition and Development, 13, 7339-7342. https://doi.org/10.18697/ajfand.56.11545

Mwadalu, R., \& Mwangi, M. (2013). The potential role of sorghum in enhancing food security in semi-arid eastern Kenya: A review. Journal of Applied Biosciences, 71(1), 5786-5799. https://doi.org/10.4314/jab.v71i1.98826

Nagel, M., Kodde, J., Pistrick, S., Mascher, M., Börner, A., \& Groot, S. P. C. (2016). Barley seed aging: Genetics behind the dry elevated pressure of oxygen aging and moist controlled deterioration. Frontiers in Plant Science, 7. https://doi.org/10.3389/fpls.2016.00388

Ng'uni, D., Geleta, M., Hofvander, P., Fatih, M., \& Bryngelsson, T. (2012). Comparative genetic diversity and nutritional quality variation among some important Southern African sorghum accessions (Sorghum bicolor L. Moench). Australian Journal of Crop Science, 6(1), 56-64. Retrieved from https://www.researchgate.net/publication/259752716

Ochieng, L. A., Mathenge, P. W., \& Muasya, R. M. (2011). A survey of on-farm seed production practices of sorghum (Sorghum bicolor L. Moench) in Bomet district of Kenya, African Journal of Food, Agriculture, Nutrition and Development, 11(5), 5232-5253. https://doi.org/10.4314/ajfand.v11i5.70448

Rao, P. S., Vinutha, K. S., Kumar, G. S., Chiranjeevi, T., Uma, A., Lal, P., \& Jose, S. (2016). Sorghum: A multipurpose bioenergy crop. Sorghum: State of the art and future perspectives, Agronmonogr, 58. https://doi.org/10.2134/agronmonogr58.2014.0074

Sahu, A. K., Sahu, B., Soni, A., \& Naithani, S. C. (2017). Active oxygen species metabolism in neem (Azadirachta indica) seeds exposed to natural ageing and controlled deterioration. Acta Physiologiae Plantarum, 39(9). https://doi.org/10.1007/s11738-017-2494-6

Sano, N., \& Rajjou, L. (2020). Lost in Translation: Physiological Roles of Stored mRNAs in Seed Germination. Plants (Basel), 9(3). https://doi.org/10.3390/plants9030347

Shisanya, C. A. Jaetzold R. Schmidt, H., \& Hornetz, B. (2006). Natural conditions and farm management information, vol. II, part B: Central Kenya, 1-493. Retrieved from https://library.wur.nl/isric/fulltext/isricu_i00023897_001.pdf

Swigonova, Z., Lai, J., Ma, J., Ramakrishna, W., Llaca, V., Bennetzen, J. L., \&Messing, J. (2004). Close split of sorghum and maize genome progenitors. Genome Research, 14, 1916-1923. https://doi.org/10.1101/gr.2332504

Swinkels, R. A., Franzel, S., Shepherd, K. D., Ohlsson, E., \& Ndufa, J. K. (1997). The economics of short rotation improved fallows: evidence from areas of high population density in Western Kenya. Agricultural 
systems, 55, 99-121. https://doi.org/10.1016/S0308-521X(96)00098-4

Verma, R., Kumar, R., \& Nath, A. (2018). Drought Resistance Mechanism and Adaptation to Water Stress in Sorghum [Sorghum bicolor (L.) Moench]. International Journal of Bio-resource and Stress Management, 9(1), 167-172. https://doi.org/10.23910/IJBSM/2018.9.1.3C0472

Zhu, S. Y., Hong, D. L., Yao, J., Zhang, X. L., \& Luo, T. K., (2010). Improving germination, seedling establishment and biochemical characters of aged hybrid rice seed by priming with KNO3 + PVA. African Journal of Agricultural Research, 5(1), 078-083. Retrieved from http://www.academicjournals.org/AJAR

\section{Copyrights}

Copyright for this article is retained by the author(s), with first publication rights granted to the journal.

This is an open-access article distributed under the terms and conditions of the Creative Commons Attribution license (http://creativecommons.org/licenses/by/3.0/). 